Pacific

Journal of

Mathematics

\title{
FOUR-DIMENSIONAL OSSERMAN METRICS OF NEUTRAL SIGNATURE
}

Eduardo García-Río, Peter B. Gilkey,

M. ELENA VÁZqueZ-ABAL AND RAMÓN VÁZquEZ-LORENZO 


\title{
FOUR-DIMENSIONAL OSSERMAN METRICS OF NEUTRAL SIGNATURE
}

\author{
Eduardo García-Río, Peter B. Gilkey, \\ M. Elena VÁZQueZ-Abal AND RAmón VÁZQUEZ-LoRENZO
}

\begin{abstract}
In the algebraic context, we show null Osserman, spacelike Osserman, and timelike Osserman are equivalent conditions for a model of signature $(2,2)$. We also classify the null Jordan Osserman models of signature $(2,2)$. In the geometric context, we show that a pseudo-Riemannian manifold with this signature is null Jordan Osserman if and only if either it has constant sectional curvature or it is locally a complex space form.
\end{abstract}

\section{Introduction}

Let $\mathcal{M}:=(M, g)$ be a pseudo-Riemannian manifold. We say a tangent vector $v$ is spacelike, timelike, or null if $g(v, v)>0$, if $g(v, v)<0$, or if $g(v, v)=0$, respectively. Geometric properties derived from conditions on spacelike, timelike, and null vectors can have quite different meanings. For instance, the conditions of spacelike, timelike, and null geodesic completeness are nonequivalent and independent. Although spacelike and timelike conditions can sometimes become equivalent (for example, as they concern boundedness conditions on the sectional curvature), they can be quite different from similar null conditions, which are sometimes related to the conformal geometry of the manifold.

Let $R(x, y):=\nabla_{x} \nabla_{y}-\nabla_{y} \nabla_{x}-\nabla_{[x, y]}$ be the curvature operator of $\mathcal{M}$. The associated Jacobi operator $\mathscr{F}_{R}(x): y \rightarrow R(y, x) x$ encodes much of the manifold's geometric information. The rescaling property $\mathscr{F}_{R}(\lambda v)=\lambda^{2} \mathscr{F}_{R}(v)$ plays a crucial role. Let $S^{ \pm}(M)$ be the unit sphere bundles of spacelike and timelike unit tangent vectors in $M$, and let $N(M)$ be the null cone of nonzero null vectors. One says that $\mathcal{M}$ is spacelike Osserman if the eigenvalues of $\mathscr{F}_{R}$ are constant on $S^{+}(\mathcal{M})$; one says instead timelike if they are constant on $S^{-}(\mathcal{M})$. Normalizing the length

MSC2000: primary 53C20, 53C50; secondary 53B30.

Keywords: spacelike, timelike and null Jacobi operator, Osserman and Jordan Osserman metric, neutral signature $(2,2)$.

García-Río, Vázquez-Abal, and Vázquez-Lorenzo are supported by projects MTM2006-01432 and PGIDIT06PXIB207054PR (Spain). Gilkey is partially supported by the Max Planck Institute in the Mathematical Sciences (Leipzig, Germany) and by project MTM2006-01432 (Spain). 
of the tangent vector to be \pm 1 takes into account the above scaling of the Jacobi operator. Perhaps surprisingly, spacelike Osserman and timelike Osserman are equivalent conditions [García-Río et al. 1999; Gilkey 2001].

We say that $\mathcal{M}$ is null Osserman if the eigenvalues of $\mathscr{F}_{R}$ are constant on the null cone $N(\mathcal{M})$; with this definition, if $\mathcal{M}$ is null Osserman, then necessarily $\mathscr{F}_{R}(v)$ is nilpotent if $v \in N(\mathcal{M})$ and $\mathscr{F}_{R}(v)$ has only the eigenvalue 0 . Any spacelike or timelike Osserman manifold is necessarily null Osserman; the converse can fail in general - see for example [García-Río et al. 1997] in the Lorentzian setting.

The Jordan normal form plays a crucial role in the higher signature setting a self-adjoint linear transformation need not be determined by its eigenvalues if the metric in question is indefinite. One says that $M$ is spacelike, timelike, or null Jordan Osserman if the Jordan normal form of $\mathscr{F}_{R}(\cdot)$ is constant on $S^{+}(\mathcal{M})$, on $S^{-}(\mathcal{M})$, or on $N(\mathcal{M})$, respectively. It is known from [Gilkey 2001; Gilkey and Ivanova 2002; 2001] that spacelike and timelike Jordan Osserman are inequivalent conditions; further neither necessarily implies the null Jordan Osserman condition.

In this paper, we concentrate on the 4-dimensional setting. Chi [1988] showed that any Riemannian Osserman 4-manifold is locally isometric to a 2-point homogeneous space; from later work [Blažić et al. 1997; García-Río et al. 1997], it follows that any Lorentzian 4-manifold has constant sectional curvature. However the situation is much more complicated in neutral signature $(2,2)$; there exist many examples of nonsymmetric Osserman pseudo-Riemannian manifolds of neutral signature — see [Díaz-Ramos et al. 2006b] and [García-Río et al. 1998]. Despite the results of [Alekseevsky et al. 1999; Blažić et al. 2001; Díaz-Ramos et al. 2006a; García-Río and Vázquez-Lorenzo 2001], it is still an open problem to completely describe 4-dimensional Osserman metrics of neutral signature.

It is convenient to work algebraically. Let $V$ be a finite-dimensional real vector space that is equipped with a nondegenerate symmetric bilinear form $\langle\cdot, \cdot\rangle$ of signature $(p, q)$. Let $A \in \otimes^{4}\left(V^{*}\right)$ be an algebraic curvature tensor on $V$, that is, a tensor that has the symmetries of the Riemann curvature tensor:

$$
\begin{aligned}
& A(x, y, z, v)=-A(y, x, z, v)=A(z, v, x, y), \\
& A(x, y, z, v)+A(y, z, x, v)+A(z, x, y, v)=0 .
\end{aligned}
$$

This defines a model $\mathfrak{M}:=(V,\langle\cdot, \cdot\rangle, A)$. We often prove results on the algebraic level (that is, for models), and then obtain corresponding conclusions in the geometric context. The notions spacelike unit vector, timelike unit vector, null vector, Jacobi operator, and so on extend naturally to this setting.

1.1. Null Osserman algebraic curvature tensors. Henceforth, suppose $\langle\cdot, \cdot\rangle$ is an inner product of signature $(2,2)$ on a 4-dimensional real vector space $V$. Fix 
an orientation of $V$, and let $\mathscr{B}=\left\{e_{1}, e_{2}, e_{3}, e_{4}\right\}$ be an oriented orthonormal basis for $V$, where $e_{1}$ and $e_{2}$ are timelike and where $e_{3}$ and $e_{4}$ are spacelike.

At the algebraic level, in signature $(2,2)$ the conditions spacelike Osserman, timelike Osserman, spacelike Jordan Osserman and timelike Jordan Osserman are equivalent to the condition that $\mathfrak{M}$ is Einstein and self-dual with respect to a suitably chosen local orientation [Alekseevsky et al. 1999; García-Río et al. 2002]. In Section 2, we will establish Theorem 1.2, which shows that these conditions are also equivalent to null Osserman:

Theorem 1.2. Let $\mathfrak{M}$ be a model of neutral signature $(2,2)$. Then the following conditions are equivalent:

(1) $\mathfrak{M}$ is spacelike Osserman.

(2) $\mathfrak{M}$ is timelike Osserman.

(3) $\mathfrak{M}$ is spacelike Jordan Osserman.

(4) $\mathfrak{M}$ is timelike Jordan Osserman.

(5) $\mathfrak{M}$ is Einstein and self-dual for a suitably chosen local orientation.

(6) $\mathfrak{M}$ is null Osserman.

Remark 1.3. The action of homothety on the null vectors is a central one in this subject. With our definition, it is immediate that $\mathfrak{M}=(V,\langle\cdot, \cdot\rangle, A)$ is null Osserman implies that 0 is the only eigenvalue of $\mathscr{F}_{A}$ on $N(V,\langle\cdot, \cdot\rangle)$. There is, although, an alternative, and different, formulation. One says that $\mathfrak{M}$ is projectively null Osserman if either $\mathfrak{M}$ is null Osserman or if given $0 \neq n_{1}, n_{2} \in N(V,\langle\cdot, \cdot\rangle)$, there is a nonzero constant $\lambda$ such that $\operatorname{Spec}\left(\mathscr{F}_{A}\left(n_{1}\right)\right)=\lambda \operatorname{Spec}\left(\mathscr{F}_{A}\left(n_{2}\right)\right)$. We refer to [Brozos-Vázquez et al. 2008] for related work; we only introduce this concept for the sake of completeness as it plays no role in our development.

1.4. Null Jordan Osserman algebraic curvature tensors. Two algebraic curvature tensors will play a distinguished role. If $\Psi$ is an skew-adjoint endomorphism of $V$, define the associated algebraic curvature tensor $A^{\Psi}$ by setting

$$
A^{\Psi}(x, y, z, v):=\langle\Psi y, z\rangle\langle\Psi x, v\rangle-\langle\Psi x, z\rangle\langle\Psi y, v\rangle-2\langle\Psi x, y\rangle\langle\Psi z, v\rangle .
$$

Such tensors span the linear space of all algebraic curvature tensors [Fiedler 2002].

The sectional curvature of a nondegenerate 2-plane $\pi=\operatorname{Span}\{x, y\}$ is given by

$$
K_{A}(\pi):=\frac{A(x, y, y, x)}{\langle x, x\rangle\langle y, y\rangle-\langle x, y\rangle\langle x, y\rangle} ;
$$

$A$ has constant sectional curvature $\kappa_{0}$ if and only if $A=\kappa_{0} A^{0}$, where $A^{0}$ is the algebraic curvature tensor of constant sectional curvature +1 defined by

$$
A^{0}(x, y, z, v):=\langle y, z\rangle\langle x, v\rangle-\langle x, z\rangle\langle y, v\rangle .
$$


We note that (1-1) and (1-2) imply that

$$
\mathscr{F}_{A^{\Psi}}(x): y \rightarrow 3\langle y, \Psi x\rangle \Psi x \text { and } \mathscr{F}_{A^{0}}(x): y \rightarrow\langle x, x\rangle y-\langle x, y\rangle x .
$$

Assume that $\Psi$ is skew-adjoint. We say $\Psi$ is an orthogonal complex structure if $\Psi^{2}=-\mathrm{id}$ and say $\Psi$ is an adapted paracomplex structure if $\Psi^{2}=\mathrm{id}$. We say that a triple of skew-adjoint operators $\left\{\Psi_{1}, \Psi_{2}, \Psi_{3}\right\}$ is a paraquaternionic structure if $\Psi_{1}^{2}=-\mathrm{id}, \Psi_{2}^{2}=\mathrm{id}, \Psi_{3}^{2}=\mathrm{id}$, and if $\Psi_{i} \Psi_{j}+\Psi_{j} \Psi_{i}=0$ for $i \neq j$. We can define a paraquaternionic structure by setting

$$
\begin{aligned}
& \Psi_{1} e_{1}=-e_{2}, \quad \Psi_{1} e_{2}=e_{1}, \quad \Psi_{1} e_{3}=e_{4}, \quad \Psi_{1} e_{4}=-e_{3}, \\
& \Psi_{2} e_{1}=e_{3}, \quad \Psi_{2} e_{2}=e_{4}, \quad \Psi_{2} e_{3}=e_{1}, \quad \Psi_{2} e_{4}=e_{2} \text {, } \\
& \Psi_{3} e_{1}=e_{4}, \quad \Psi_{3} e_{2}=-e_{3}, \quad \Psi_{3} e_{3}=-e_{2}, \quad \Psi_{3} e_{4}=e_{1} .
\end{aligned}
$$

Note that $\Psi_{3}=\Psi_{1} \Psi_{2}$. If $\left\{\bar{\Psi}_{1}, \bar{\Psi}_{2}, \bar{\Psi}_{3}\right\}$ is another paraquaternionic structure on $V$, there is an isometry $\phi$ of $V$ such that $\phi^{*} \bar{\Psi}_{1}=\Psi_{1}, \phi^{*} \bar{\Psi}_{2}=\Psi_{2}$, and $\phi^{*} \bar{\Psi}_{3}= \pm \Psi_{3}$; this slight sign ambiguity plays no role in our constructions.

Let $x$ be a spacelike or timelike vector. Then there is an orthogonal direct sum decomposition $V=\mathbb{R} x \oplus x^{\perp}$. Since $\mathscr{F}_{A}(x) x=0, \mathscr{F}_{A}(x)$ preserves $x^{\perp}$. There are four different possibilities that describe the Jordan normal form of $\mathscr{F}_{A}(x)$ restricted to $x^{\perp}$ (see [Blažić et al. 2001; García-Río et al. 2002] for further details):

$$
\begin{aligned}
& \left(\begin{array}{ccc}
\alpha & 0 & 0 \\
0 & \beta & 0 \\
0 & 0 & \gamma
\end{array}\right),\left(\begin{array}{ccc}
\alpha & -\beta & 0 \\
\beta & \alpha & 0 \\
0 & 0 & \gamma
\end{array}\right),\left(\begin{array}{ccc}
\beta & 0 & 0 \\
0 & \alpha & 0 \\
0 & 1 & \alpha
\end{array}\right),\left(\begin{array}{lll}
\alpha & 0 & 0 \\
1 & \alpha & 0 \\
0 & 1 & \alpha
\end{array}\right) . \\
& \text { Type Ia Type Ib Type II Type III }
\end{aligned}
$$

Type Ia corresponds to a diagonalizable operator, Type Ib to an operator with a complex eigenvalue and Type II (respectively Type III) to a double (respectively triple) root of the minimal polynomial of the operator. If $\mathfrak{M}$ is spacelike, timelike, or null Osserman, then the Jordan normal form of $\mathscr{F}_{A}$ is constant on the spacelike and timelike unit vectors, and we classify $A$ according to the four types above. In Section 3, we construct, up to isomorphism, all the spacelike Jordan Osserman algebraic curvature tensors and perform the analysis necessary to establish the following classification result:

Theorem 1.5. Let $\mathfrak{M}:=(V,\langle\cdot, \cdot\rangle, A)$ be a model of signature $(2,2)$. Then $\mathfrak{M}$ is null Jordan Osserman if and only if $A$ is of Type Ia and one of the following holds:

(1) There exists a constant $\kappa_{0}$ such that $A=\kappa_{0} A^{0}$.

(2) There exists constants $\kappa_{0}$ and $\kappa_{J}$ with $\kappa_{J} \neq 0$ such that $A=\kappa_{0} A^{0}+\kappa_{J} A^{J}$, where $J$ is an orthogonal complex structure on $V$.

(3) There exists a constant $\kappa_{P} \neq 0$ such that $A=\kappa_{P} A^{P}$, where $P$ is an adapted paracomplex structure on $V$. 
(4) There exist constants $\kappa_{1}, \kappa_{2}, \kappa_{3}$ such that $\kappa_{2} \kappa_{3}\left(\kappa_{2}+\kappa_{1}\right)\left(\kappa_{3}+\kappa_{1}\right)>0$, such that the associated eigenvalues $\left\{3 \kappa_{1},-3 \kappa_{2},-3 \kappa_{3}\right\}$ are all distinct, and such that $A=\kappa_{1} A^{\Psi_{1}}+\kappa_{2} A^{\Psi_{2}}+\kappa_{3} A^{\Psi_{3}}$, where $\left(\Psi_{1}, \Psi_{2}, \Psi_{3}\right)$ is a paraquaternionic structure on $V$.

Remark 1.6. The inequality $\kappa_{2} \kappa_{3}\left(\kappa_{2}+\kappa_{1}\right)\left(\kappa_{3}+\kappa_{1}\right)>0$ is equivalent to the cross ratio satisfying

$$
\left(0, \kappa_{1},-\kappa_{3},-\kappa_{2}\right)=\frac{\kappa_{3}\left(\kappa_{2}+\kappa_{1}\right)}{\kappa_{2}\left(\kappa_{3}+\kappa_{1}\right)}>0 .
$$

Let $\mathbb{S}^{2}$ be the unit sphere in $\mathbb{R}^{3}$. This inequality is equivalent to the fact that the set of points $\left(0,-\kappa_{3},-\kappa_{2}\right)$ and $\left(\kappa_{1},-\kappa_{3},-\kappa_{2}\right)$ give (via the stereographic projection) the corresponding circles in $\mathbb{S}^{2}$ the same orientation [Marden 2007].

1.7. Null Jordan Osserman manifolds. We characterize those neutral signature 4manifolds that are null Jordan Osserman; null Osserman and null Jordan Osserman are not equivalent conditions, as the analysis of Section 3.7 shows. We say that $M$ is locally a complex space form if it is an indefinite Kähler manifold of constant holomorphic sectional curvature. In Section 4, we will use Theorem 1.5 to establish the following geometric result:

Theorem 1.8. If $\mathcal{M}$ is a connected pseudo-Riemannian manifold of neutral signature $(2,2)$, then $\mathcal{M}$ is null Jordan Osserman if and only if either $\mathcal{M}$ has constant sectional curvature or $\mathcal{M}$ is locally a complex space form.

Remark 1.9. There is another family of Osserman 4-manifolds with diagonalizable Jacobi operator, namely, the paracomplex space forms [Blažić et al. 2001]. Although the geometry of complex and paracomplex space forms is very similar, the Jordan-Osserman condition distinguishes them. To our knowledge, this is the first algebraic curvature condition that distinguishes these two geometries.

\section{Null Osserman models of signature $(2,2)$}

We work in the algebraic context to prove Theorem 1.2. Here is a brief outline to this section. Previous work establishes that parts (1)-(5) are equivalent. In Section 2.1, we introduce notation and show that spacelike Osserman models are null Osserman and that null Osserman models are Einstein. Thus to complete the proof, it suffices to show null Osserman models are self-dual or anti-self-dual. In Section 2.3, we examine Einstein models. Lemma 2.4 describes the Weyl curvature operators in that setting, and Lemma 2.5 gives an alternative characterization of self-duality for an Einstein model. We use Lemma 2.5 to complete the proof of Theorem 1.2 in Section 2.6. 
2.1. Notation. Let $\mathfrak{M}:=(V,\langle\cdot, \cdot\rangle, A)$ be a neutral signature 4-dimensional model. We use the inner product to raise indices and to define an associated Jacobi operator $\mathscr{F}_{A}$, which is characterized by the identity $\left\langle\mathscr{F}_{A}(x) y, z\right\rangle=A(y, x, x, z)$. Let $\mathscr{B}=\left\{e_{1}, e_{2}, e_{3}, e_{4}\right\}$ be an oriented orthonormal basis for $V$ as in Section 1.1. Let $g_{i j}:=\left\langle e_{i}, e_{j}\right\rangle$, and let $g^{i j}$ be the inverse matrix. The associated Ricci tensor $\rho_{A}$, the scalar curvature $\tau_{A}$, and the Weyl tensor $W_{A}$ are then defined by setting

$$
\begin{array}{r}
\rho_{A}(x, y):=\sum_{i, j=1}^{4} g^{i j} A\left(e_{i}, x, y, e_{j}\right), \quad \tau_{A}:=\sum_{i, j=1}^{4} g^{i j} \rho_{A}\left(e_{i}, e_{j}\right), \\
W_{A}(x, y, z, v):=A(x, y, z, v)+\frac{1}{6} \tau_{A}(\langle y, z\rangle\langle x, v\rangle-\langle x, z\rangle\langle y, v\rangle) \\
-\frac{1}{2}\left(\rho_{A}(y, z)\langle x, v\rangle-\rho_{A}(x, z)\langle y, v\rangle\right. \\
\left.+\rho_{A}(x, v)\langle y, z\rangle-\rho_{A}(y, v)\langle x, z\rangle\right) .
\end{array}
$$

Let $A_{i j k l}=A_{i j k l}^{\mathscr{B}}:=A\left(e_{i}, e_{j}, e_{k}, e_{l}\right)$ denote the components of $A$ with respect to $\mathscr{B}$, where $1 \leq i, j, k, l \leq 4$; we drop the dependence on $\mathscr{B}$ from the notation when there is no danger of confusion. Let $\left\{e^{1}, \ldots, e^{4}\right\}$ be the dual basis for $V^{*}$. The Hodge operator $\star: \Lambda^{p}\left(V^{*}\right) \rightarrow \Lambda^{4-p}\left(V^{*}\right)$ is characterized by the identity

$$
\phi_{p} \wedge \star \theta_{p}=\left\langle\phi_{p}, \theta_{p}\right\rangle e^{1} \wedge e^{2} \wedge e^{3} \wedge e^{4} .
$$

Thus,

$$
\begin{aligned}
& \star\left(e^{1} \wedge e^{2}\right)=e^{3} \wedge e^{4}, \quad \star\left(e^{1} \wedge e^{3}\right)=e^{2} \wedge e^{4}, \quad \star\left(e^{1} \wedge e^{4}\right)=-e^{2} \wedge e^{3}, \\
& \star\left(e^{2} \wedge e^{3}\right)=-e^{1} \wedge e^{4}, \quad \star\left(e^{2} \wedge e^{4}\right)=e^{1} \wedge e^{3}, \quad \star\left(e^{3} \wedge e^{4}\right)=e^{1} \wedge e^{2} .
\end{aligned}
$$

A crucial feature of 4-dimensional geometry now enters. Since $\star^{2}=\mathrm{id}$, the Hodge star induces a splitting $\Lambda^{2}\left(V^{*}\right)=\Lambda^{+} \oplus \Lambda^{-}$of the space of 2-forms, where

$$
\Lambda^{+}=\left\{\alpha \in \Lambda^{2}: \star \alpha=\alpha\right\} \quad \text { and } \Lambda^{-}=\left\{\alpha \in \Lambda^{2}: \star \alpha=-\alpha\right\}
$$

denote the spaces of self-dual and anti-self-dual two-forms. We have orthonormal bases $\left\{E_{1}^{\mp}, E_{2}^{\mp}, E_{3}^{\mp}\right\}$ for $\Lambda^{\mp}$ that are given by

$$
\begin{aligned}
& E_{1}^{\mp}=\frac{1}{\sqrt{2}}\left(e^{1} \wedge e^{2} \mp e^{3} \wedge e^{4}\right), \quad E_{2}^{\mp}=\frac{1}{\sqrt{2}}\left(e^{1} \wedge e^{3} \mp e^{2} \wedge e^{4}\right), \\
& E_{3}^{\mp}=\frac{1}{\sqrt{2}}\left(e^{1} \wedge e^{4} \pm e^{2} \wedge e^{3}\right),
\end{aligned}
$$

where the induced inner product on $\Lambda^{\mp}$ has signature $(2,1)$ :

$$
\left\langle E_{1}^{\mp}, E_{1}^{\mp}\right\rangle=1, \quad\left\langle E_{2}^{\mp}, E_{2}^{\mp}\right\rangle=-1, \quad\left\langle E_{3}^{\mp}, E_{3}^{\mp}\right\rangle=-1 .
$$

Let $W_{A}^{\mp}$ be the restriction of $W_{A}$ to the spaces $\Lambda^{\mp}$, that is, $W_{A}^{\mp}: \Lambda^{\mp} \rightarrow \Lambda^{\mp}$, where $W_{A}$ also stands for the associated Weyl curvature operator on $\Lambda^{2}$. We say $\mathfrak{M}$ is self-dual if $W_{A}^{-}=0$ and anti-self-dual if $W_{A}^{+}=0$.

Lemma 2.2. Let $\mathfrak{M}=(V,\langle\cdot, \cdot\rangle, A)$ be a model of signature $(2,2)$. 
(1) If $\mathfrak{M}$ is spacelike Osserman, then $\mathfrak{M}$ is null Osserman.

(2) If $\mathfrak{M}$ is null Osserman, then $\mathfrak{M}$ is Einstein.

Proof. Suppose first $\mathfrak{M}$ is spacelike Osserman. Set $T_{j}(v):=\operatorname{Tr}\left\{\mathscr{F}_{A}(v)^{j}\right\}$. Since the eigenvalues of $\mathscr{F}_{A}$ are constant on $S^{+}(V,\langle\cdot, \cdot\rangle)$, there are constants $c_{j}$ such that $T_{j}(v)=c_{j}$ for $v \in S^{+}(V,\langle\cdot, \cdot\rangle)$. It follows since $T_{j}(\lambda v)=\lambda^{2 j} T_{j}(v)$ that $T_{j}(v)=c_{j}\langle v, v\rangle^{j}$ for $v$ spacelike. Since the spacelike vectors form an open subset of $V$, this polynomial identity holds for all $v \in V$. Thus, in particular, $T_{j}(v)=0$ if $v \in N(V,\langle\cdot, \cdot\rangle)$. This implies that 0 is the only eigenvalue of $\mathscr{F}_{A}(v)$ and shows $\mathfrak{M}$ is null Osserman.

Now suppose $\mathfrak{M}$ is null Osserman. Let $s_{1}$ and $s_{2}$ be spacelike unit vectors. We may choose a unit timelike vector $t$ that is perpendicular to $s_{1}$ and $s_{2}$. Let $n_{i}^{ \pm}:=s_{i} \pm t$ be null vectors. Thus $0=\operatorname{Tr}\left(\mathscr{F}_{A}\left(n_{i}^{ \pm}\right)\right)=\rho_{A}\left(n_{i}^{ \pm}, n_{i}^{ \pm}\right)$, and

$$
0=\rho_{A}\left(s_{i} \pm t, s_{i} \pm t\right)=\rho_{A}\left(s_{i}, s_{i}\right)+\rho_{A}(t, t) \pm 2 \rho_{A}\left(s_{i}, t\right) .
$$

This implies $\rho_{A}\left(s_{i}, t\right)=0$ and $\rho_{A}\left(s_{i}, s_{i}\right)+\rho_{A}(t, t)=0$; in particular, one has $\rho_{A}\left(s_{1}, s_{1}\right)=-\rho_{A}(t, t)=\rho_{A}\left(s_{2}, s_{2}\right)$. Therefore, after rescaling, there is a constant $c$ such that $\rho_{A}(s, s)=c\langle s, s\rangle$ for every spacelike vector $s$; this polynomial identity then continues to hold for all $s \in V$. Polarizing this identity then yields $\rho_{A}=c\langle\cdot, \cdot\rangle$, and hence $\mathfrak{M}$ is Einstein.

\subsection{The Weyl tensor for an Einstein algebraic curvature tensor. Let}

$$
\begin{aligned}
& \sigma_{1}=2 A_{1212}+3 \varepsilon A_{1234}+A_{1313}+A_{1414}, \\
& \sigma_{2}=A_{1212}+2 A_{1313}+3 \varepsilon A_{1324}-A_{1414}, \\
& \sigma_{3}=A_{1212}+3 \varepsilon A_{1234}-A_{1313}-3 \varepsilon A_{1324}+2 A_{1414} .
\end{aligned}
$$

Then we have an immediate lemma:

Lemma 2.4. If $\mathfrak{M}$ is Einstein, then the self-dual Weyl curvature operator $W_{A}^{+}$(in which $\varepsilon=1)$ and the anti-self-dual Weyl curvature operator $W_{A}^{-}($in which $\varepsilon=-1)$ are given by

$$
\left(\begin{array}{ccc}
\sigma_{1} / 3 & A_{1213}+\varepsilon A_{1224} & A_{1214}-\varepsilon A_{1223} \\
-A_{1213}-\varepsilon A_{1224} & -\sigma_{2} / 3 & -A_{1314}+\varepsilon A_{1323} \\
-A_{1214}+\varepsilon A_{1223} & -A_{1314}+\varepsilon A_{1323} & -\sigma_{3} / 3
\end{array}\right) .
$$

The next observation is of interest in its own right:

Lemma 2.5. If $\mathfrak{M}$ is Einstein, then the model $\mathfrak{M}$ is anti-self-dual if and only if $A_{1214}^{\mathscr{B}}-A_{1223}^{\mathscr{B}}=0$ for every oriented orthonormal frame $\mathscr{B}$.

Proof. If $\mathfrak{M}$ is anti-self-dual, we set $\varepsilon=1$ in Lemma 2.4 to see $A_{1214}^{\mathscr{B}}-A_{1223}^{\mathscr{P}}=0$. Conversely, suppose $A_{1214}^{\mathscr{R}}-A_{1223}^{\mathscr{P}}=0$ for every $\mathscr{B}$. Define a new basis $\tilde{\mathscr{B}}$ by 
setting $\tilde{e}_{1}=e_{1}, \tilde{e}_{2}=e_{2}, \tilde{e}_{3}=e_{4}$, and $\tilde{e}_{4}=-e_{3}$. We then have

$$
0=-A_{1214}^{\tilde{\Re_{B}}}+A_{1223}^{\tilde{\mathscr{B}}_{3}}=A_{1213}^{\mathscr{\Re}}+A_{1224}^{\mathscr{\Re}} \text {. }
$$

Next, define $\tilde{\mathscr{P}}$ by setting $\tilde{e}_{1}=e_{1}, \tilde{e}_{2}=\cosh \theta e_{2}+\sinh \theta e_{3}, \tilde{e}_{3}=\sinh \theta e_{2}+\cosh \theta e_{3}$, and $\tilde{e}_{4}=e_{4}$. This yields the relation

$$
0=-A_{1214}^{\tilde{\mathscr{B}_{1}}}+A_{1223}^{\tilde{\mathscr{P}}}=\cosh \theta\left(-A_{1214}^{\mathscr{P}}+A_{1223}^{\mathscr{P}}\right)+\sinh \theta\left(-A_{1314}^{\mathscr{乃}}+A_{1323}^{\mathscr{\Re}}\right) .
$$

This shows $-A_{1314}^{\mathscr{B}}+A_{1323}^{\mathscr{P}}=0$. Thus, by Lemma 2.4,

$$
W_{A}^{+}=\frac{1}{3}\left(\begin{array}{ccc}
\sigma_{1}^{\Re} & 0 & 0 \\
0 & -\sigma_{2}^{\mathscr{B}} & 0 \\
0 & 0 & -\sigma_{3}^{\Re}
\end{array}\right) .
$$

Setting the $\tilde{e}_{i}$ as before yields bases for $\Lambda^{ \pm}$in the form

$$
\tilde{E}_{1}^{ \pm}=\cosh \theta E_{1}^{ \pm}+\sinh \theta E_{2}^{ \pm}, \quad \tilde{E}_{2}^{ \pm}=\cosh \theta E_{2}^{ \pm}+\sinh \theta E_{1}^{ \pm}, \quad \tilde{E}_{3}^{ \pm}=E_{3}^{ \pm} .
$$

We may compute

$$
\begin{aligned}
W_{A}^{+} \tilde{E}_{1}^{+} & =\sigma_{1}^{\tilde{\mathscr{P}}} \tilde{E}_{1}^{+}=\sigma_{1}^{\tilde{\mathscr{P}}}\left(\cosh \theta E_{1}^{+}+\sinh \theta E_{2}^{+}\right) \\
& =W_{A}^{+}\left(\cosh \theta E_{1}^{+}+\sinh \theta E_{2}^{+}\right)=\sigma_{1}^{\mathscr{\Re}} \cosh \theta E_{1}^{+}-\sigma_{2}^{\mathscr{P}} \sinh \theta E_{2}^{+} .
\end{aligned}
$$

This shows $\sigma_{1}^{\tilde{\mathscr{B}}}=\sigma_{1}^{\mathscr{B}}=-\sigma_{2}^{\mathscr{P}}$. A similar argument applied to the basis $\tilde{e}_{1}=e_{1}$, $\tilde{e}_{2}=\cosh \theta e_{2}+\sinh \theta e_{4}, \tilde{e}_{3}=e_{3}$, and $\tilde{e}_{4}=\sinh \theta e_{2}+\cosh \theta e_{4}$ yields $\sigma_{1}^{\mathscr{B}}=-\sigma_{3}^{\mathscr{B}}$. Since $\sigma_{1}^{\mathscr{P}}-\sigma_{2}^{\mathscr{B}}-\sigma_{3}^{\mathscr{B}}=0$, it now follows that $W_{A}^{+}=0$.

2.6. Proof of Theorem 1.2. Let $\mathfrak{M}$ be a null Osserman model. By Lemma 2.2, $\mathfrak{M}$ is Einstein. We complete the proof of Theorem 1.2 by showing $\mathfrak{M}$ is self-dual or anti-self-dual. Suppose the contrary and argue for a contradiction. As $\mathfrak{M}$ is null Osserman, $\mathscr{F}_{A}$ is nilpotent, so the characteristic polynomial has $p_{\lambda}\left(\mathscr{F}_{A}(u)\right)=\lambda^{4}$. Let

$$
\begin{aligned}
\mathscr{E}_{1}:= & A_{1212}+2 A_{1214}-2 A_{1223}+2 A_{1234}-A_{1324}+A_{1414}, \\
Q(a, b):= & \left.A_{1212}-2 A_{1214}-2 A_{1223}-2 A_{1234}+A_{1324}+A_{1414}\right) a^{4} \\
& +\left(A_{1212}+2 A_{1214}+2 A_{1223}-2 A_{1234}+A_{1324}+A_{1414}\right) b^{4} \\
& +2\left(A_{1212}+2 A_{1313}-3 A_{1324}-A_{1414}\right) a^{2} b^{2} \\
& +4\left(A_{1213}-A_{1224}-A_{1314}-A_{1323}\right) a^{3} b \\
& +4\left(A_{1213}-A_{1224}+A_{1314}+A_{1323}\right) a b^{3} .
\end{aligned}
$$

If we take $u=a e_{1}+b e_{2}+a e_{3}+b e_{4}$, then $\lambda^{4}=p_{\lambda}\left(\mathscr{F}_{A}(u)\right)=\lambda^{2}\left(\lambda^{2}-Q(a, b) \mathscr{E}_{1}\right)$. As $p_{\lambda}\left(\mathscr{F}_{A}(u)\right)=\lambda^{4}$, either $Q(a, b)=0$ or $\mathscr{E}_{1}=0$. If we suppose that $\mathscr{E}_{1} \neq 0$, then 
$Q(a, b)$ vanishes identically for all $a, b$. This leads to the relations

$$
\begin{aligned}
A_{1213}-A_{1224} & =0, \quad A_{1214}+A_{1223}=0, \quad A_{1314}+A_{1323}=0, \\
A_{1234}+A_{1313}-2 A_{1324}-A_{1414} & =0, \quad A_{1212}+2 A_{1313}-3 A_{1324}-A_{1414}=0 .
\end{aligned}
$$

From this, we see that the matrix in Lemma 2.4 vanishes for $\varepsilon=-1$. This means that the anti-self-dual Weyl curvature operator $W_{A}^{-}$vanishes, so $\mathfrak{M}$ is self-dual, which is a contradiction. Thus for any oriented orthonormal frame, we have

$$
0=A_{1212}+2 A_{1214}-2 A_{1223}+2 A_{1234}-A_{1324}+A_{1414} .
$$

Setting $\tilde{e}_{1}=-e_{1}, \tilde{e}_{2}=e_{2}, \tilde{e}_{3}=e_{3}$, and $\tilde{e}_{4}=-e_{4}$ yields

$$
0=A_{1212}-2 A_{1214}+2 A_{1223}+2 A_{1234}-A_{1324}+A_{1414} .
$$

Subtracting (2-2) from (2-1) then yields the relation $0=-A_{1214}+A_{1223}$. We may now use Lemma 2.5 to complete the proof of Theorem 1.2.

\section{Proof of Theorem 1.5}

Here is a brief outline of this section. In Section 3.1, we construct, up to isomorphism, all spacelike Jordan Osserman models of signature $(2,2)$. In the remainder of Section 3, we analyze each possible Jordan normal form in some detail using the classification of (1-5). Sections 3.5-3.8 deal with Type Ia models. In Section 3.5 we study the case when all the eigenvalues are equal; this gives rise to Theorem 1.5(1). In Section 3.6, we study the case of two equal spacelike eigenvalues, and in Section 3.7, we study equal timelike and spacelike eigenvalues; these involve parts (2) and (3) of Theorem 1.5, respectively. In Section 3.8, we study Type Ia models with distinct eigenvalues; this leads to Theorem 1.5(4). We complete the proof of Theorem 1.5 by showing the remaining types do not give rise to null Jordan Osserman models. We study Type Ib models in Section 3.9, Type II models in Section 3.10, and Type III models in Section 3.11.

3.1. Spacelike Jordan Osserman models. We use the ansatz from [Gilkey and Ivanova 2001]. Let $\left\{\Psi_{1}, \Psi_{2}, \Psi_{3}\right\}$ be the paraquaternionic structure given in (1-4). Let $\xi_{i j} \in \mathbb{R}$ for $1 \leq i \leq j \leq 3$, and let $\kappa_{0} \in \mathbb{R}$ be given. Let

$$
\begin{aligned}
A_{\kappa_{0}, \xi}:=\kappa_{0} A^{0}+\frac{1}{3} \xi_{11} A^{\Psi_{1}}+\frac{1}{3} \xi_{22} A^{\Psi_{2}}+\frac{1}{3} \xi_{33} A^{\Psi_{3}} & \\
+\frac{1}{3} \xi_{12} A^{\Psi_{1}+\Psi_{2}}+\frac{1}{3} \xi_{13} A^{\Psi_{1}+\Psi_{3}}+\frac{1}{3} \xi_{23} A^{\Psi_{2}+\Psi_{3}}, & \Phi_{\kappa_{0}, \xi}:=\kappa_{0} \mathrm{id}+\left(\begin{array}{rrr}
\xi_{11}+\xi_{12}+\xi_{13} & -\xi_{12} & -\xi_{13} \\
\xi_{12}-\xi_{22}-\xi_{12}-\xi_{23} & -\xi_{23} \\
\xi_{13} & -\xi_{23}-\xi_{33}-\xi_{13}-\xi_{23}
\end{array}\right) .
\end{aligned}
$$

Lemma 3.2. Adopt the notation established above. Let $\mathfrak{M}_{\kappa_{0}, \xi}:=\left(V,\langle\cdot, \cdot\rangle, A_{\kappa_{0}, \xi}\right)$. 
(1) If $x \in S^{ \pm}(V,\langle\cdot, \cdot\rangle)$, then $\mathscr{F}_{A_{\kappa_{0}, \xi}}(x)$ is conjugate to the matrix $\pm \mathscr{F}_{\kappa_{0}, \xi}$.

(2) The model $\mathfrak{M}_{\kappa_{0}, \xi}$ is spacelike and timelike Jordan Osserman.

(3) Let $\mathfrak{M}_{i}=\left(V,\langle\cdot, \cdot\rangle, A_{i}\right)$ be spacelike Osserman models of signature $(2,2)$. If $\mathscr{F}_{A_{1}}(x)$ is conjugate to $\mathscr{F}_{A_{2}}(x)$ for some $x \in S^{ \pm}(V,\langle\cdot, \cdot\rangle)$, then there exists an isometry $\phi$ of $(V,\langle\cdot, \cdot\rangle)$ such that $\phi^{*} A_{2}=A_{1}$.

Remark 3.3. Any self-adjoint map of a signature $(2,1)$ vector space is conjugate to $\mathscr{F}_{\kappa_{0}, \xi}$ for some $\left\{\kappa_{0}, \xi\right\}$, so every spacelike Osserman model of signature $(2,2)$ is isomorphic to one given by (3-1).

Proof. We suppose $x$ is a spacelike unit vector; the timelike case is similar. Let $f_{1}:=\Psi_{1} x, f_{2}:=\Psi_{2} x$, and $f_{3}:=\Psi_{3} x$. Then $\left\{f_{1}, f_{2}, f_{3}\right\}$ is an orthonormal basis of signature $(+,-,-)$ for $x^{\perp}$. Let $\mathscr{F}:=\mathscr{F}_{A_{\kappa_{0}, \xi}}(x)$. We use (1-3) to see that

$$
\begin{aligned}
& \mathscr{g} f_{1}=\left(\kappa_{0}+\xi_{11}+\xi_{12}+\xi_{13}\right) f_{1}+\xi_{12} f_{2}+\xi_{13} f_{3}, \\
& \mathscr{f} f_{2}=-\xi_{12} f_{1}+\left(\kappa_{0}-\xi_{22}-\xi_{12}-\xi_{23}\right) f_{2}-\xi_{23} f_{3}, \\
& \mathscr{g} f_{3}=-\xi_{13} f_{1}-\xi_{23} f_{2}+\left(\kappa_{0}-\xi_{33}-\xi_{13}-\xi_{23}\right) f_{3} .
\end{aligned}
$$

Part (1) now follows; part (2) follows from part (1). Suppose that $\mathfrak{M}$ is a Type Ia spacelike Osserman model, so $\mathscr{F}_{A}(x)=\operatorname{diag}[\alpha, \beta, \gamma]$ for any $x$ in $S^{+}(V,\langle\cdot, \cdot\rangle)$; choose the notation so $\operatorname{Ker}\left(\mathscr{F}_{A}(x)-\alpha\right.$ id $)$ is spacelike. It then follows from the discussion in [Blažić et al. 2001; García-Río et al. 2002] that there is an orthonormal basis $\mathscr{B}$ such that the nonzero components of the curvature tensor are

$$
\begin{array}{ll}
A_{1221}=A_{4334}=\alpha, & A_{1331}=A_{2442}=-\beta, \\
A_{1441}=A_{3223}=-\gamma, & A_{1234}=(-2 \alpha+\beta+\gamma) / 3, \\
A_{1423}=(\alpha+\beta-2 \gamma) / 3, & A_{1342}=(\alpha-2 \beta+\gamma) / 3 .
\end{array}
$$

Similar forms exist for the other types of (1-5). Thus the Jordan normal form of $\mathscr{F}_{A}(x)$ determines $A$ up to the action of $\mathrm{O}(2,2)$. Part (3) follows.

We immediately have this:

Lemma 3.4. A null Osserman model $\mathfrak{M}$ of signature $(2,2)$ is null Jordan Osserman if and only if the functions $\operatorname{Rank}\left\{\mathscr{F}_{A}(\cdot)\right\}$ and $\operatorname{Rank}\left\{\mathscr{F}_{A}(\cdot)^{2}\right\}$ are constant on $N(V,\langle\cdot, \cdot\rangle)$.

3.5. Type Ia with all eigenvalues equal: $\boldsymbol{\alpha}=\boldsymbol{\beta}=\boldsymbol{\gamma}$. We set $A=\kappa_{0} A^{0}$. By Lemma 3.2, the Jordan normal form is given by $\operatorname{diag}\left[\kappa_{0}, \kappa_{0}, \kappa_{0}\right]$. If $v$ belongs to $N(V,\langle\cdot, \cdot\rangle)$, then $\mathscr{F}_{A}(v) y=-\kappa_{0}\langle v, y\rangle v$, and hence $\mathfrak{M}$ is null Jordan Osserman. 
3.6. Type Ia with two equal spacelike eigenvalues: $\beta=\gamma$ and $\alpha \neq \beta$. Let $J$ be an orthogonal almost complex structure on $V$, and let $A=\kappa_{0} A^{0}+\kappa_{J} A^{J}$. The Jordan normal form is then given by $\operatorname{diag}\left[\kappa_{0}+3 \kappa_{J}, \kappa_{0}, \kappa_{0}\right]$, which has the desired form for suitably chosen $\kappa_{0}$ and $\kappa_{J}$ with $\kappa_{J} \neq 0$. Let $v \in N(V,\langle\cdot, \cdot\rangle)$. We have

$$
\mathscr{F}_{A}(v) y=-\kappa_{0}\langle v, y\rangle v+3 \kappa_{J}\langle y, J v\rangle J v .
$$

Because $J^{2}=-\mathrm{id}$, the vectors $v$ and $J v$ are linearly independent. We note that $\langle v, v\rangle=\langle v, J v\rangle=\langle J v, J v\rangle=0$. Consequently $\mathscr{F}_{A}(v) v=\mathscr{F}_{A}(v) J v=0$. Since $v^{\perp}$ and $J v^{\perp}$ are distinct 3-dimensional subspaces, we can choose $y$ so $\langle v, y\rangle=1$ and $\langle J v, y\rangle=0$. It now follows that $\mathscr{F}_{A}(v) y=-\kappa_{0} v$, while $\mathscr{f}_{A}(v) J y=3 \kappa_{J} J v$. Thus $\mathscr{F}_{A}(v)$ has rank 2 and $\mathscr{F}_{A}(v)^{2}=0$. This implies $A$ is null Jordan Osserman.

\subsection{Type Ia with equal timelike and spacelike eigenvalues: $\alpha=\beta$ and $\beta \neq \gamma$.} Let $A=\kappa_{0} A^{0}+\kappa_{P} A^{P}$, where $\kappa_{P} \neq 0$ and where $P$ is an adapted paracomplex structure; the Jordan normal form is then given by $\operatorname{diag}\left[\kappa_{0}, \kappa_{0}-3 \kappa_{P}, \kappa_{0}\right]$, which has the desired form for suitably chosen parameters. If $v \in N(V,\langle\cdot, \cdot\rangle)$, then

$$
\mathscr{E}_{A}(v) y=-\kappa_{0}\langle v, y\rangle v+3 \kappa_{P}\langle y, P v\rangle P v .
$$

If $\kappa_{0}=0, \mathfrak{M}$ is null Jordan Osserman. Suppose $\kappa_{0} \neq 0$. If $v=e_{1}+P e_{1}$, then $P v=v$, so $\operatorname{Rank}\left\{\mathscr{F}_{A}(v)\right\} \leq 1$. On the other hand, if $v=e_{1}+e_{4}$, then $v$ and $P v$ are linearly independent, so $\operatorname{Rank}\left\{\mathscr{F}_{A}(v)\right\}=2$ and $\mathfrak{M}$ is not null Jordan Osserman.

3.8. Type Ia with three distinct eigenvalues. We set $A:=\sum_{i} \kappa_{i} A^{\Psi_{i}}$, where the triple $\left\{\Psi_{1}, \Psi_{2}, \Psi_{3}\right\}$ is the paraquaternionic structure of (1-4); the Jordan normal form is given by $\operatorname{diag}\left[3 \kappa_{1},-3 \kappa_{2},-3 \kappa_{3}\right]$, which has the desired form for suitably chosen parameters with

$$
\kappa_{1}+\kappa_{2} \neq 0, \quad \kappa_{1}+\kappa_{3} \neq 0, \quad \kappa_{2}-\kappa_{3} \neq 0 .
$$

Let $\tilde{e} \in S^{+}(V,\langle\cdot, \cdot\rangle)$, let $V_{+}:=\operatorname{Span}\left\{\tilde{e}, \Psi^{1} \tilde{e}\right\}$, and let $V_{-}=V_{+}^{\perp}=\operatorname{Span}\left\{\Psi_{2} \tilde{e}, \Psi_{3} \tilde{e}\right\}$. We then have an orthogonal direct sum decomposition $V=V_{-} \oplus V_{+}$, where $V_{+}$ is spacelike and $V_{-}$is timelike. Decompose $v \in N(V,\langle\cdot, \cdot\rangle)$ as $v=\lambda\left(e_{+}+e_{-}\right)$, where $e_{ \pm} \in V_{ \pm}$. Let $\mathfrak{M}$ be spacelike Osserman. We have $\mathscr{F}_{A}(v)=\lambda^{2} \mathscr{F}_{A}\left(e_{+}+e_{-}\right)$. Since $\mathscr{F}_{A}(v)$ is nilpotent, $\mathscr{F}_{A}(v)$ and $\mathscr{F}_{A}\left(e_{+}+e_{-}\right)$have the same Jordan normal form. Thus we may safely take $\lambda=1$, so $v=e_{+}+e_{-}$. Set $e=e_{+}$and expand $e_{-}=\cos \theta \Psi_{2} e+\sin \theta \Psi_{3} e$. This expresses

$$
v=e+\cos \theta \Psi_{2} e+\sin \theta \Psi_{3} e \quad \text { for } e \in S^{+}(V,\langle\cdot, \cdot\rangle) .
$$


We use the relations $\Psi_{1} \Psi_{2}=\Psi_{3}, \Psi_{1} \Psi_{3}=-\Psi_{2}$, and $\Psi_{2} \Psi_{3}=-\Psi_{1}$ to see that

$$
\Psi_{1} v=0+\Psi_{1} e-\sin \theta \Psi_{2} e+\cos \theta \Psi_{3} e,
$$

$$
\Psi_{2} v=\cos \theta e-\sin \theta \Psi_{1} e+\Psi_{2} e+0 \text {, }
$$$$
\Psi_{3} v=\sin \theta e+\cos \theta \Psi_{1} e+0+\Psi_{3} e,
$$

so that $0=\Psi_{1} v+\sin \theta \Psi_{2} v-\cos \theta \Psi_{3} v$. Thus the vectors $\left\{\Psi_{1} v, \Psi_{2} v, \Psi_{3} v\right\}$ span a 2-dimensional subspace. Since $\left\langle\Psi_{i} v, \Psi_{j} v\right\rangle=0, \operatorname{Span}\left\{\Psi_{i} v\right\} \subset \operatorname{Ker}\left\{\mathscr{F}_{A}(v)\right\}$. Since Range $\left\{\mathscr{F}_{A}(v)\right\} \subset \operatorname{Span}\left\{\Psi_{i} v\right\}$,

$$
\operatorname{Rank}\left\{\mathscr{F}_{A}(v)\right\} \leq 2 \text { and } \mathscr{F}_{A}(v)^{2}=0 .
$$

Note that $\left\{e, \Psi_{1} e, \Psi_{2} v, \Psi_{3} v\right\}$ is a basis for $V$. Let $\pi_{+}$denote orthogonal projection on $V_{+}=\operatorname{Span}\left\{e, \Psi_{1} e\right\}$. Since $\pi_{+}$is injective on $\operatorname{Range}\left\{\mathscr{F}_{A}(v)\right\} \subset \operatorname{Span}\left\{\Psi_{2} v, \Psi_{3} v\right\}$,

$$
r(v):=\operatorname{dim} \operatorname{Range}\left\{\mathscr{F}_{A}(v)\right\}=\operatorname{dim}\left(\operatorname{Span}\left\{\pi_{+} \mathscr{F}_{A}(v) e, \pi_{+} \mathscr{\mathscr { F }}_{A}(v) \Psi_{1} e\right\}\right) .
$$

By (3-2) and the linear dependency it contains,

$$
\begin{aligned}
\mathscr{F}_{A}(v) e= & 3 \kappa_{2} \cos \theta \Psi_{2} v+3 \kappa_{3} \sin \theta \Psi_{3} v, \\
\mathscr{F}_{A}(v) \Psi_{1} e= & 3 \kappa_{1} \Psi_{1} v-3 \kappa_{2} \sin \theta \Psi_{2} v+3 \kappa_{3} \cos \theta \Psi_{3} v, \\
\pi_{+} \mathscr{F}_{A}(v) e= & 3\left(\kappa_{2} \cos \theta(\cos \theta)+\kappa_{3} \sin \theta(\sin \theta)\right) e \\
& +3\left(\kappa_{2} \cos \theta(-\sin \theta)+\kappa_{3} \sin \theta(\cos \theta)\right) \Psi_{1} e, \\
\pi_{+} \mathscr{F}_{A}(v) \Psi_{1} e= & 3\left(-\kappa_{2} \sin \theta(\cos \theta)+\kappa_{3} \cos \theta(\sin \theta)\right) e \\
& +3\left(\kappa_{1}-\kappa_{2} \sin \theta(-\sin \theta)+\kappa_{3} \cos \theta(\cos \theta)\right) \Psi_{1} e .
\end{aligned}
$$

This leads to a coefficient matrix for $\pi_{+} \mathscr{F}_{A}(v)$ on $V_{+}$given by

$$
\mathscr{C}_{A}(\theta)=3\left(\begin{array}{rr}
\kappa_{2} \cos ^{2} \theta+\kappa_{3} \sin ^{2} \theta & \left(-\kappa_{2}+\kappa_{3}\right) \sin \theta \cos \theta \\
\left(-\kappa_{2}+\kappa_{3}\right) \sin \theta \cos \theta & \kappa_{1}+\kappa_{2} \sin ^{2} \theta+\kappa_{3} \cos ^{2} \theta
\end{array}\right) .
$$

We compute

$$
\begin{aligned}
\frac{1}{9} \operatorname{det}\left(\mathscr{C}_{A}\right)(\theta)= & \kappa_{1} \kappa_{2} \cos ^{2} \theta+\kappa_{2}^{2} \cos ^{2} \theta \sin ^{2} \theta+\kappa_{2} \kappa_{3} \cos ^{4} \theta \\
& +\kappa_{1} \kappa_{3} \sin ^{2} \theta+\kappa_{2} \kappa_{3} \sin ^{4} \theta+\kappa_{3}^{2} \sin ^{2} \theta \cos ^{2} \theta \\
& -\kappa_{2}^{2} \sin ^{2} \theta \cos ^{2} \theta-\kappa_{3}^{2} \sin ^{2} \theta \cos ^{2} \theta+2 \kappa_{2} \kappa_{3} \sin ^{2} \theta \cos ^{2} \theta \\
= & \kappa_{1} \kappa_{2} \cos ^{2} \theta+\kappa_{1} \kappa_{3} \sin ^{2} \theta+\kappa_{2} \kappa_{3} \\
= & \left(\kappa_{1}+\kappa_{3}\right) \kappa_{2} \cos ^{2} \theta+\left(\kappa_{1}+\kappa_{2}\right) \kappa_{3} \sin ^{2} \theta .
\end{aligned}
$$

Observe that $\kappa_{2} \kappa_{3}=0$ implies that $\operatorname{det}\left(\mathscr{C}_{A}\right)(\theta)$ vanishes for some $\theta$, and thus $\mathfrak{M}$ is not null Jordan Osserman. Hence, since $\left(\kappa_{1}+\kappa_{3}\right) \kappa_{2}$ and $\left(\kappa_{1}+\kappa_{2}\right) \kappa_{3}$ are nonzero, $\operatorname{det}\left(\mathscr{C}_{A}\right)(\theta)$ never vanishes, or equivalently $\mathfrak{M}$ is null Jordan Osserman, if and only if these two real numbers have the same sign, that is, $\kappa_{2} \kappa_{3}\left(\kappa_{1}+\kappa_{3}\right)\left(\kappa_{1}+\kappa_{2}\right)>0$. 
3.9. Type Ib models. Let $b \neq 0$. We take a curvature tensor of the form

$$
A=\frac{1}{3}\left((a-b) A^{\Psi_{1}}+(-b-a) A^{\Psi_{2}}+b A^{\Psi_{1}+\Psi_{2}}+c A^{\Psi_{3}}\right) .
$$

Proceeding as in the previous case, we have for any $e \in S^{+}(V,\langle\cdot, \cdot\rangle)$ that

$$
\begin{aligned}
\mathscr{F}_{A}(x) y & =\left\langle\left(a \Psi_{1}+b \Psi_{2}\right) x, y\right\rangle \Psi_{1} x+\left\langle\left(b \Psi_{1}-a \Psi_{2}\right) x, y\right\rangle \Psi_{2} x+c\left\langle\Psi_{3} x, y\right\rangle \Psi_{3} x, \\
\mathscr{J}_{A}(e) \Psi_{1} e & =a \Psi_{1} e+b \Psi_{2} e, \mathscr{F}_{A}(e) \Psi_{2} e=-b \Psi_{1} e+a \Psi_{2} e, \mathscr{F}_{A}(e) \Psi_{3} e=-c \Psi_{3} e .
\end{aligned}
$$

Thus $\mathfrak{M}:=(V,\langle\cdot, \cdot\rangle, A)$ is Type $\mathrm{Ib}$ and any Type $\mathrm{Ib}$ model is isomorphic to $\mathfrak{M}$ for suitably chosen parameters. As in Section 3.8, put $v=e+\cos \theta \Psi_{2} e+\sin \theta \Psi_{3} e$. We compute

$$
\begin{aligned}
\mathscr{F}_{A}(v) e= & b \cos \theta \Psi_{1} v-a \cos \theta \Psi_{2} v+c \sin \theta \Psi_{3} v, \\
\mathscr{F}_{A}(v) \Psi_{1} e= & (a-b \sin \theta) \Psi_{1} v+(b+a \sin \theta) \Psi_{2} v+c \cos \theta \Psi_{3} v, \\
\pi_{+} \mathscr{I}_{A}(v) e= & (-a \cos \theta(\cos \theta)+c \sin \theta(\sin \theta)) e \\
& +(b \cos \theta-a \cos \theta(-\sin \theta)+c \sin \theta(\cos \theta)) \Psi_{1} e, \\
\pi_{+} \mathscr{J}_{A}(v) \Psi_{1} e= & ((b+a \sin \theta)(\cos \theta)+c \cos \theta(\sin \theta)) e \\
& +((a-b \sin \theta)+(b+a \sin \theta)(-\sin \theta)+c \cos \theta(\cos \theta)) \Psi_{1} e .
\end{aligned}
$$

The coefficient matrix for $\pi_{+} \mathscr{F}_{A}(v)$ on $V_{+}$is then given by

$$
\mathscr{C}_{A}(\theta)=\left(\begin{array}{rr}
-a \cos ^{2} \theta+c \sin ^{2} \theta & b \cos \theta+(a+c) \sin \theta \cos \theta \\
b \cos \theta+(a+c) \sin \theta \cos \theta & -2 b \sin \theta+(a+c) \cos ^{2} \theta
\end{array}\right) .
$$

We have $\operatorname{det}\left(\mathscr{C}_{A}\right)(\pi / 2)=-2 b c$ and $\operatorname{det}\left(\mathscr{C}_{A}\right)(-\pi / 2)=2 b c$. If $c \neq 0$, then these signs differ and hence $\operatorname{det}\left(\mathscr{C}_{A}\right)(\theta)=0$ for some $-\pi / 2<\theta<\pi / 2$ and $\mathfrak{M}$ is not null Jordan Osserman. If $c=0$, then $\operatorname{det}\left(\mathscr{C}_{A}\right)(\pi / 2)=0$ and $\operatorname{det}\left(\mathscr{C}_{A}\right)(0)=-a^{2}-b^{2} \neq 0$ and again $\mathfrak{M}$ is not null Jordan Osserman.

3.10. Type II models. We approach this case directly. Let $\mathfrak{M}=(V,\langle\cdot, \cdot\rangle, A)$ be a model of signature $(2,2)$, where $A$ is a Type II algebraic curvature tensor. Then the analysis of [Blažić et al. 2001; García-Río et al. 2002] shows there exists an orthonormal basis $\left\{e_{1}, e_{2}, e_{3}, e_{4}\right\}$ for $V$ such that the nonvanishing components of $A$ are

$$
\begin{array}{ll}
A_{1221}=A_{4334}= \pm\left(\alpha-\frac{1}{2}\right), & A_{1224}=A_{1334}= \pm \frac{1}{2}, \\
A_{1331}=A_{4224}=\mp\left(\alpha+\frac{1}{2}\right), & A_{2113}=A_{2443}=\mp \frac{1}{2}, \\
A_{1234}=\left( \pm\left(-\alpha+\frac{3}{2}\right)+\beta\right) / 3, & A_{1423}=2( \pm \alpha-\beta) / 3, \\
A_{1342}=\left( \pm\left(-\alpha-\frac{3}{2}\right)+\beta\right) / 3, & A_{1441}=A_{3223}=-\beta .
\end{array}
$$


Let $u=e_{2}-e_{3}$ and let $v=e_{2}+e_{3}$. Then

$$
\mathscr{F}_{A}(u)=\left(\begin{array}{rrrr}
0 & 0 & 0 & 0 \\
0 & \beta & \beta & 0 \\
0 & -\beta & -\beta & 0 \\
0 & 0 & 0 & 0
\end{array}\right) \quad \text { and } \mathscr{F}_{A}(v)=\left(\begin{array}{rrrr} 
\pm 2 & 0 & 0 & \mp 2 \\
0 & \beta & -\beta & 0 \\
0 & \beta & -\beta & 0 \\
\pm 2 & 0 & 0 & \mp 2
\end{array}\right) .
$$

If $\beta=0$, then $r(u)=0$ and $r(v)=1$; if $\beta \neq 0$, then $r(u)=1$ and $r(v)=2$. Thus $\mathfrak{M}$ is not null Jordan Osserman.

3.11. Type III models. For $\mathfrak{M}$ of this type, there exists by [Blažić et al. 2001; García-Río et al. 2002] an orthonormal basis $\left\{e_{1}, e_{2}, e_{3}, e_{4}\right\}$ for $V$ such that the nonvanishing components of $A$ are

$$
\begin{aligned}
& A_{1221}=A_{4334}=\alpha, \quad A_{1331}=A_{4224}=-\alpha, \\
& A_{1441}=A_{3223}=-\alpha, \\
& A_{2114}=A_{2334}=-\sqrt{2} / 2, \quad A_{3114}=-A_{3224}=\sqrt{2} / 2, \\
& A_{1223}=A_{1443}=A_{1332}=-A_{1442}=\sqrt{2} / 2 .
\end{aligned}
$$

Let $u=e_{2}-e_{3}$ and $v=e_{2}+e_{3}$. Then

$$
\mathscr{F}_{A}(u)=\left(\begin{array}{rrrr}
0 & -\sqrt{2} & -\sqrt{2} & 0 \\
-\sqrt{2} & \alpha & \alpha & \sqrt{2} \\
\sqrt{2} & -\alpha & -\alpha & -\sqrt{2} \\
0 & -\sqrt{2} & -\sqrt{2} & 0
\end{array}\right) \quad \text { and } \mathscr{F}_{A}(v)=\left(\begin{array}{cccc}
0 & 0 & 0 & 0 \\
0 & \alpha & -\alpha & 0 \\
0 & \alpha & -\alpha & 0 \\
0 & 0 & 0 & 0
\end{array}\right) \text {. }
$$

It now follows that $r(u)=2$ while $r(v) \leq 1$ and hence $\mathfrak{M}$ is not null Jordan Osserman. This completes the proof of Theorem 1.5.

\section{The proof of Theorem 1.8}

Let $\mathcal{M}$ be a null Jordan Osserman manifold of signature $(2,2)$. First note that, by Theorem 1.5, $\mathcal{M}$ has Type Ia. Results of [Blažić et al. 2001] then show that $\mathcal{M}$ either has constant sectional curvature, is locally isometric to a complex space form, or is locally isometric to a paracomplex space form. Since the curvature tensor of a paracomplex space form of constant paraholomorphic sectional curvature $\kappa$ satisfies

$$
R(x, y) z=\frac{1}{4} \kappa\left(R^{0}(x, y) z-R^{J}(x, y) z\right),
$$

this is ruled out by Theorem 1.5, thus proving Theorem 1.8. 


\section{References}

[Alekseevsky et al. 1999] D. Alekseevsky, N. Blažić, N. Bokan, and Z. Rakić, "Self-duality and pointwise Osserman manifolds", Arch. Math. (Brno) 35:3 (1999), 193-201. MR 2000j:53094 Zbl 1054.53082

[Blažić et al. 1997] N. Blažić, N. Bokan, and P. Gilkey, "A note on Osserman Lorentzian manifolds", Bull. London Math. Soc. 29:2 (1997), 227-230. MR 97m:53111 Zbl 0865.53018

[Blažić et al. 2001] N. Blažić, N. Bokan, and Z. Rakić, "Osserman pseudo-Riemannian manifolds of signature (2, 2)”, J. Aust. Math. Soc. 71:3 (2001), 367-395. MR 2002g:53119 Zbl 1011.53049

[Brozos-Vázquez et al. 2008] M. Brozos-Vázquez, P. Gilkey, S. Nikčević, and U. Simon, "Projectively Osserman manifolds", Publ. Math. Debrecen 72:3-4 (2008), 359-370. MR 2406926 Zbl 05367462

[Chi 1988] Q.-S. Chi, "A curvature characterization of certain locally rank-one symmetric spaces", J. Differential Geom. 28:2 (1988), 187-202. MR 90a:53060 Zbl 0654.53053

[Díaz-Ramos et al. 2006a] J. C. Díaz-Ramos, E. García-Río, and R. Vázquez-Lorenzo, "Fourdimensional Osserman metrics with nondiagonalizable Jacobi operators", J. Geom. Anal. 16:1 (2006), 39-52. MR 2006m:53107 Zbl 1105.53052

[Díaz-Ramos et al. 2006b] J. C. Díaz-Ramos, E. García-Río, and R. Vázquez-Lorenzo, "New examples of Osserman metrics with nondiagonalizable Jacobi operators", Differential Geom. Appl. 24:4 (2006), 433-442. MR 2007h:53106 Zbl 1099.53047

[Fiedler 2002] B. Fiedler, "Determination of the structure of algebraic curvature tensors by means of Young symmetrizers", Sém. Lothar. Combin. 48 (2002), Art. B48d. MR 2004d:53018

[García-Río and Vázquez-Lorenzo 2001] E. García-Río and R. Vázquez-Lorenzo, "Four-dimensional Osserman symmetric spaces", Geom. Dedicata 88:1-3 (2001), 147-151. MR 2002k:53137 Zbl 1008.53023

[García-Río et al. 1997] E. García-Río, D. N. Kupeli, and M. E. Vázquez-Abal, "On a problem of Osserman in Lorentzian geometry”, Differential Geom. Appl. 7:1 (1997), 85-100. MR 98a:53099 Zbl 0880.53017

[García-Río et al. 1998] E. García-Río, M. E. Vázquez-Abal, and R. Vázquez-Lorenzo, "Nonsymmetric Osserman pseudo-Riemannian manifolds", Proc. Amer. Math. Soc. 126:9 (1998), 27712778. MR 98m:53089 Zbl 0939.53014

[García-Río et al. 1999] E. García-Río, D. N. Kupeli, M. E. Vázquez-Abal, and R. Vázquez-Lorenzo, "Affine Osserman connections and their Riemann extensions", Differential Geom. Appl. 11:2 (1999), 145-153. MR 2000i:53030 Zbl 0940.53017

[García-Río et al. 2002] E. García-Río, D. N. Kupeli, and R. Vázquez-Lorenzo, Osserman manifolds in semi-Riemannian geometry, Lecture Notes in Mathematics 1777, Springer, Berlin, 2002. MR 2003e:53052 Zbl 1005.53040

[Gilkey 2001] P. B. Gilkey, Geometric properties of natural operators defined by the Riemann curvature tensor, World Scientific Publishing, River Edge, NJ, 2001. MR 2002k:53052 Zbl 1007.53001

[Gilkey and Ivanova 2001] P. Gilkey and R. Ivanova, "The Jordan normal form of Osserman algebraic curvature tensors”, Results Math. 40:1-4 (2001), 192-204. MR 2002j:53093 Zbl 0999.53014

[Gilkey and Ivanova 2002] P. B. Gilkey and R. Ivanova, "Spacelike Jordan Osserman algebraic curvature tensors in the higher signature setting", pp. 179-186 in Differential geometry (Valencia, 2001), edited by O. Gil-Medrano and V. Miquel, World Scientific Publishing, River Edge, NJ, 2002. MR 2003f:53125 Zbl 1031.53037 
[Marden 2007] A. Marden, Outer circles: An introduction to hyperbolic 3-manifolds, Cambridge University Press, 2007. MR 2008i:57001 Zbl 1149.57030

Received April 3, 2008. Revised September 15, 2009.

EDUARDO GARCÍA-RÍO

DEPARTMENT OF GEOMETRY AND TOPOLOGY

FACULTY OF MATHEMATICS

University of SANTIAGo DE COMPOSTEla

15782 SANTIAGo DE COMPOSTELA

SPAIN

eduardo.garcia.rio@usc.es

Peter B. Gilkey

DEPARTMENT OF MATHEMATICS

UNIVERSITY OF OREGON

EUGENE, OR 97403

UNITED STATES

gilkey@uoregon.edu

M. ElENA VÁZQUEZ-ABAL

DEPARTMENT OF GEOMETRY AND TOPOLOGY

FACULTY OF MATHEMATICS

UNIVERSITY OF SANTIAGO DE COMPOSTELA

15782 SANTIAGo DE CoMpostela

SPAIN

elena.vazquez.abal@usc.es

RAMÓN VÁZQUEZ-LORENZO

DEPARTMENT OF GEOMETRY AND TOPOLOGY

FACULTY OF MATHEMATICS

University OF SANTIAGO DE COMPOSTElA

15782 SANTIAGo DE COMPOSTELA

SPAIN

ravazlor@edu.xunta.es 